\title{
LES ASPECTS JURIDIQUES ET FINANCIERS DE LA COOPÉRATION DES VILLES SUR LES AIRES MÉTROPOLITAINES EN POLOGNE
}

\section{Les observations préliminaires}

Les formes d'organisation et juridique de la coopération des collectivités territoriales et la portée des tâches prises en charge par celles-ci, sont les deux circonstances qui peuvent influencer les règles de réalisation de la gestion financière pour les aires métropolitaines. Dans la législation polonaise, la notion d'aire métropolitaine n'a pas été définie de façon précise, toutefois, conformément aux dispositions de la loi du 27 mars 2003 sur la planification et l'aménagement du territoire', elle est entendue comme une structure spatiale, composée d'une grande ville et des terres environnantes fonctionnelles étroitement liés à cette ville (les villes, les communes ou les districts directement adjacent à la ville); c'est la structure créée dans le concept de l'aménagement du territoire dans le pays. Pour l'aire métropolitaine, la diétine de voïvodie adopte le plan d'occupation des sols de l'aire métropolitaine comme la partie du plan d'occupation des sols de la voïvodie, dans lequel on prend en compte les résultats de la stratégie de développement de la voïvodie et dans lequel on détermine, entre autres, les zones difficiles, ainsi que les règles pour les aménagements. Au cours de la formulation et la mise en œuvre de la politique du développement, la voïvodie qui est, en Pologne, une collectivité territoriale au niveau régional désigné pour conduire une politique moderne correspondant aux normes en vigueur dans l'Union européenne, collabore avec les collectivités locales à l'intérieur de la voïvodie, avec les organismes chargés de représenter les intérêts des entreprises commerciales, industrielles et de service, avec les associations professionnelles et avec l'administration central (en particulier avec 
Les aspects juridiques et financiers de la coopération des villes sur les aires...

voïvode), et enfin avec d'autres voïvodies, les organisations non gouvernementales, les universités ainsi que les unités de recherche ${ }^{2}$.

Dans ce sens, les aires métropolitaines sont seulement une entité de planification (spatiale). En décidant de mettre en place les aires métropolitaines, il faut définir cette notion et il faut prendre en compte leur différenciation spatiale. Il faudra également décrire l'ampleur des tâches et développer des outils pour les coordonner. Dans le projet de révision de loi sur la planification et l'aménagement du territoire, on a proposé la définition de «l'aire métropolitaine» comme « L'espace de grande ville où les grandes villes mutuellement dépendantes et leur environnement directement associé d'un point de vue fonctionnel, étant créé dans le concept de l'aménagement du territoire du pays, conformément à une division territoriale de base de l'État en districts et qui représente un complexe habité par plus de 500 mille personnes, réunissant les institutions de la coopération internationale ».

Les aires métropolitaines peuvent fonctionner sous des formes diverses. A titre d'exemple, on peut indiquer que la ville de Barcelone est une commune divisée en 10 quartiers; autour de cette ville s'étend l'espace métropolitain qui comprend 35 municipalités. Jusqu'à 1997 son fonctionnement était assuré par la société « Métropole de Barcelone » puis, après sa dissolution, il a été créé trois associations à but non lucratif, dont les tâches et les zones de fonctionnement sont maintenant différentes. Dans un autre point de vue, la ville de Londres est créée par 33 communes, organisée notamment en association qui est un groupe de pression, une société de droit commercial, qui ne reçoit aucun soutien des fonds publics ou d'agence de développement. La ville de Stockholm est organisée en commune divisée en 18 quartiers, Toronto est divisé en 44 quartiers et L'Espace du Grand Toronto (Greater Toronto Area - GTA) comprend 4 régions de 24 municipalités, tandis que Sao Paulo est une commune divisée en 31 quartiers incluant les 39 communes environnantes. Cette ville entre dans la structure de la région métropolitaine ${ }^{3}$. L'objectif principal d'une telle zone est l'organisation conjointe des transports publics, le développement régional, la planification spatiale et le logement. L'Association Régionale de la Ruhr est un autre exemple - elle fonctionne sur la base de la loi et elle couvre les villes exclues du district (11) et des districts (4) qui bordent les uns les autres. Cette association est une personne morale, elle excède le niveau local et possède ses propres revenus provenant en premier lieu des contributions de certains membres ${ }^{4}$. La loi garantit le droit à l'autodétermination et elle précise les tâches de l'association.

\footnotetext{
2 Voir l'art. 11-12a - Ustawa z dnia 5 czerwca 1998 r. o samorządzie województwa (t.j. Dz.U. z 2001 r. Nr 142, poz.1590 ze zm.).

3 www.metropolis.eu

$4 \quad$ www.rvr-online.de
} 


\section{Les formes juridiques de coopération de villes}

La nécessité de coopération entre les communes voisines qui réalisent les mêmes activités existe en Pologne depuis la remise en place de l'autonomie locale en Pologne en 1990. Cependant, jusqu'à aujourd'hui, le législateur n'a proposé aucune nouvelle idée regroupant cette coopération, tant au niveau organisationnel qu'au niveau du financement. Ses tentatives de créer un projet de loi « sur la métropole » regroupant plusieurs villes - n'a à ce jour reçu aucune suite.

La coopération des collectivités territoriales en Pologne peut prendre des formes diverses. Parmi les solutions proposées, il est possible de créer des syndicats métropolitains qui peuvent exercer en vertu de la loi, les syndicats obligatoires avec des objectifs spécifiques, dénommé les «méga-villes » qui sont divisés en quartiers, ou «les districts métropolitains" composé d'unités du même statut ${ }^{5}$. Toutefois, chacune de ces propositions est liée à la nécessité d'examiner les conséquences financières de cette réglementation et la façon d'utiliser au mieux les instruments existants pour coordonner ses tâches. La sélection des solutions choisies décide des sources potentielles de financement et également des restrictions découlant des dispositions de la Constitution et des lois en vigueur.

La possibilité de créer un syndicat de communes, afin de mener conjointement les tâches publiques, est établie par l'art. 64 alinéa 1 de la loi du 8 mars 1990 sur les municipalités de commune ${ }^{6}$. Ce syndicat ne peut être à caractère obligatoire (alinéa 4) et l'obligation de sa création ne peut être imposée que par la loi qui précise les tâches et le mode d'approbation de leur statut. En revanche, la réglementation autorisant la possibilité de créer des associations de districts avec d'autres est inclus dans l'art. 65 alinéa 1 de la loi sur la municipalité de districts ${ }^{7}$, toutefois ces associations ne peuvent pas avoir de caractère obligatoire. L'essence de l'association des collectivités locales (les associations de communes et les associations de districts) se manifeste dans le fait qu'elles sont créés par les communes ou les districts pour exercer conjointement les tâches publiques ${ }^{8}$, ce qui aura lieu lorsque, pour des raisons de leur organisation ou de leurs résultats économiques, leur activité individuel dans les communes ou les districts, seront irrationnels.

Parmi les formes juridiques de la coopération entre des collectivités territoriales, le législateur a admis la possibilité de créer une association ou une adhésion à

Voir: J. Glumińska-Pawlic: Koncepcje finansowania obszarów metropolitalnych (na przykładzie aglomeracji śląskiej). /Dans:/ Ekonomiczne i organizacyjne instrumenty wspierania rozwoju lokalnego i regionalnego, Tom 2. „Zeszyty Naukowe Uniwersytetu Szczecińskiego”, Nº 471, Szczecin 2007, p. 233-241. Ustawa z dnia 8 marca 1990 r. o samorządzie gminnym (t.j. Dz.U. z 2001 r. Nr 142, poz. 1591 ze zm.). Voir aussi: R. Mikosz: Zwiazki komunalne, porozumienia komunalne, stowarzyszenia gmin. Edition ZPP, Katowice 1991. 
l'association déjà existante avec le transfert des autorités pour l'exécution des tâches publiques pour un exercice conjoint; le contenu des dispositions en vigueur désigne clairement le champ d'application des tâches que l'association peut accomplir'. La création d'association ne fait pas obstacle à d'autres formes de coopération des collectivités locales, tels que le droit de conclure des accords sur l'exercice de fonctions publiques. Toutefois, il faut souligner qu'il existe des différences fondamentales parmi les diverses formes de coopération, et elles doivent être prises en compte au moment du choix des formes spécifiques de coopération. À moins que l'association soit une personne morale autonome et self-government et le sujet qui est créé par la volonté des parties intéressées, exprimé dans les résolutions ayant le même contenu, l'accord ne représente que le transfert des pouvoirs pour mener à bien les tâches accomplies par une ou plusieurs communes pour les autres; on exige également la volonté expresse des communes qui sont intéressées de conclure un $\operatorname{accord}^{10}$.

Prenant en compte l'expérience d'autres pays, on a proposé la création en Pologne de syndicats métropolitains avec des frontières définies par la $\mathrm{loi}^{11}$. Ces syndicats pourraient être composés de villes ayant droit de district et d'autres communes répondant aux critères fonctionnels et urbains de l'association, tel que défini par la loi. L'objectif principal de ces syndicats obligatoires pourrait être l'assurance de développement durable de tout l'espace métropolitain, grâce à la coordination et l'exécution de certaines tâches publiques, ainsi que par la mise en œuvre conjointe de projets confiés aux membres de l'association. En raison de la diversité spatiale des projets potentiels dans les aires métropolitaines, les auteurs de projets ont proposé de diversifier les tâches, en fonction des circonstances locales et des besoins locaux. Selon les types de tâches effectuées par les syndicats métropolitains agissant sur la base de la loi, les options possibles d'action concernant la coordination, la gestion et le transfert de la puissance sur la catégorie de tâches. L'association doit être créée dans un but précis et l'étendue de ses tâches pose un préalable fondamental pour déterminer le nombre et le type des collectivités territoriales qui peuvent entrer dans sa composition.

Toutefois, il convient de noter que les expériences sur le fonctionnement, en Pologne, des syndicats de communes, dont une partie est obligatoire, ne sont pas optimistes, et les vérifications qui ont été faites ont révélé de nombreuses irrégularités

9 Voir en plus: Ustawa o samorządzie powiatowym. Komentarz. /sous la red. B. Dolnicki/, Zakamycze 2005, p. 429 et suiv.

10 P. Brzezicka: Niektóre zagadnienia zawierania porozumień przez organy samorządu terytorialnego, „Samorząd Terytorialny” $n^{\circ} 3$, 2000, p. 42; P. Brzezicka: Porozumienie administracyjne - problemy węzłowe, „Państwo i Prawo" n 6, 2000, p. 43-50.

11 Voir: Założenia do projektu ustawy metropolitalnej. Projekt zespołu powołanego przez Wojewodę Śląskiego z dnia 16 maja 2007 r. Manuscrit reproduit. 
tant dans l'acquisition des revenus que les dépenses de fonds publics, la comptabilité, la gestion des marchés publics et également celle des biens ${ }^{12}$.

En l'absence de loi sur l'agglomération, les quatorze villes de la région Silésienne (les villes ayant droit de district) ont formé une association de communes, bien que cette méthode porte de nombreuses restrictions qui ont déjà révélé au cours des travaux sur la finalisation de statut de l'association, mais aussi les problèmes qui peuvent apparaitre dans un proche avenir, et qui peuvent porter atteinte à certains de ses précédemment, et en particulier de réduire les sources de son financement. L'Association Métropolitaine de Haut-Silésie est la zone avec la plus grande densité de population du pays - plus de 2 millions d'habitants et elle est un des plus grands espaces métropolitain d'Europe, qui s'étend sur environ $1500 \mathrm{~km} 2$. L'agglomération Silésienne génère plus de $9 \%$ du PIB et elle est en mesure d'affronter la concurrence d'autres métropoles pour attirer les investisseurs, tant nationaux qu'internationaux. L'Association de commune est un élément doté d'un grand potentiel de développement social et économique ; la nécessité de sa création a déjà été formulée dans le milieu des années 90. La faiblesse de la structure administrative ainsi que de la capitale, l'absence d'un centre scientifique, économique ou culturel a eu pour conséquence dans cette région, un développement beaucoup moins rapide que d'autres villes polonaises telles que Wroclaw, Poznan et Gdansk. L'Association peut devenir un partenaire dans la promotion de l'activité dans l'Union européenne, et sera responsable de la répartissions des fonds Européens d'aides aux programmes sectoriels, un point qui permettrait d'améliorer la compétitivité de Silésie par rapport aux autres métropoles, tout en réduisant la concurrence à cet égard entre elles. Conformément aux dispositions du statut de l'Association, elle a pour objet la réalisation de stratégie commune pour le développement de la région métropolitaine, afin de recueillir des fonds intérieurs ou étrangers et les ressources publiques provenant du budget de l'UE, pour améliorer l'activité sur le marché du travail, promouvoir des programmes novateurs visant à améliorer la compétitivité économique des villes et de mener à bien une politique commune parmi d'autres dans les domaines des transports locaux, y compris la gestion des moyens importants du point de vue de l'agglomération de Silésie. Dans la zone d'intérêt de l'Association sont également inclues certaines tâches dans le domaine de la protection de l'environnement, la gestion des déchets, l'eau et les déchets industrielles ainsi que l'approvisionnement en énergie.

12 Les informations sur les résultats de la gestion financière de contrôle des associations intercommunales, le Conseil national des chambres régionales des comptes, La Commission de Coordination de Contrôle, Katowice 2007. („Informacja o wynikach kontroli gospodarki finansowej związków międzygminnych”, Krajowa Rada Regionalnych Izb Obrachunkowych, Komisja Koordynacji Kontroli). 
Les aspects juridiques et financiers de la coopération des villes sur les aires...

\section{Les objectifs principaux et les tâches de métropole}

En fait, dans la discussion en cours on soulève les questions relatives aux modalités de création de régions métropolitaines en Pologne, susceptibles de concurrencer avec les régions métropolitaines de l'UE et du monde, mais aussi les questions relatives au statut: l'opportunité de créer des groupes métropolitains c'est-à-dire une institution particulière de coopération une ville avec les communes de l'agglomération, mais l'autre par rapport au syndicat de communes, ou des districts métropolitains - les unités particulières des administrations territoriale, mais en même temps les unités de base de la division territoriale et qui assurera une plus grande capacité d'accomplir leurs missions publiques, conformément à l'art. 15 et 16 de la Constitution. Il est nécessaire également de résoudre un autre problème - est-ce que l'administration du métropole devrait signifier la centralisation de certaines tâches, de compétences ainsi que de revenus des villes, des communes et des districts liées au niveau de l'agglomération ou la décentralisation de tâches métropolitaines et de recettes de l'État et de voïvodie pour le compte des autorités de la région métropolitaine.

Les tâches métropolitaines sont assimilées à des obligations de capital accomplies au niveau national ou trans-national. Le sujet métropolitain devrait en particulier:

- mettre en place une stratégie du développement et un plan de l'aménagement du territoire de l'aire métropolitaine, compatible avec la Stratégie de Développement du Pays et la conception de l'Aménagement Territoriale du Pays et réaliser ces projets à travers les programmes opérationnels,

- fixer de manière obligatoire les réseaux du transport, les réseaux d'hypermarchés et les nouveaux espaces à la colonisation massive ainsi que les zones protégées contre les bâtiments, créer et réaliser la politique métropolitaine dans le domaine de la protection des paysages et de l'environnement, établir un plan de gestion des déchets,

- voter et mettre en place la politique métropolitaine dans le domaine du transport et les programmes d'investissement de renouvellement et de l'expansion du centre de réseau TEN-Transports, en collaboration avec les programmes opérationnels nationaux et régionaux et avec les programmes de partenariat public-privé,

- gérer le réseau routier local et le trafic routier dans l'aire métropolitaine, coordonner le maintien et le développement des transports publics, y compris fixer les prix des services rendus dans l'agglomération (un billet commun) 
- maintenir - en coopération avec la police agissant sous un gestionnaire dans l'ensemble de l'aire métropolitaine - le Centre Intégrale de Gestion de la Crise en Métropole,

- promouvoir le développement de l'enseignement supérieur, des instituts scientifiques, des institutions de recherche et de développement et les divisions d'innovation des entreprises et - en consultation avec le curateur de l'éducation - établir le réseau des collèges,

- assister à la création et à l'exercice des accords intercommunaux, des sociétés et des syndicats de communes pour atteindre l'objectif commun p.ex. dans le domaine de la communication et des sujets qui misent en œuvre des projets co-financés par les fonds européens, dont les projets réalisés en vertu de partenariat public-privé,

- promouvoir les villes en organisant des événements d'importance internationale au niveau médiale et touristique et - en accord avec GUS (l'Office Générale de la Statistique) - organiser l'Office (le département) Métropolitaine de la Statistique qui étudiera la statistique de l'aire métropolitaine dans les cadres des sous-régions statistiques de NUTS-3 .

L'objectif principal de la construction et du fonctionnement de la métropole est un nouvel élan du développement et de l'amélioration de la coopération avec d'autres pays de l'UE, afin d'accroître la compétitivité, mais aussi l'amélioration de la coopération de la ville centrale de l'aire métropolitaine avec les communes dans l'agglomération; l'objectif juridique concerne l'attribution de la personnalité pour les aires métropolitaine pour mettre fin à processus de décentralisation de l'autorité publique.

L'Observatoire en Réseau de l'Aménagement du Territoire Européen (ORATE) indique en Pologne 8 régions métropolitaines, à savoir: Varsovie, Gdansk, Katowice, Cracovie, Lodz, Poznan, Szczecin et Wroclaw. Il prend en compte la population comme le critère de base (en 2006 de 725,000 à 3.065,000 d'habitants). Les critères d'ORATE sont réunis par les métropoles suivantes : Bydgoszcz-Toruń, Białystok, Lublin et Rzeszów (de 504,000 à 758,000 d'habitants). Par contre, dans la hiérarchie métropolitaine du monde on désigne:

- la métropole transnationale d'intérêt mondial (par exemple Londres),

- la métropole transnationale d'intérêt continental (par exemple Bruxelles),

- la métropole transnationale d'intérêt sub-continental (par exemple Berlin),

- la métropole nationale - les capitales des pays (par exemple Varsovie, Prague), 
- la métropole sub-national - les capitales des macro-régions (par exemple Wroclaw, Poznan).

Les aires métropolitaines doivent être compatibles avec les critères de création des sous-régions NTS-3, en tenant compte des liens sociaux, économiques, culturels et écologiques. Bien que la Nomenclature statistique des unités territoriales (NTS), adoptée par le règlement du Conseil des ministres du 14 novembre 2007 formellement doit être utile pour les objectifs statistiques, elle est également utilisée dans l'élaboration des politiques des états membres de l'Union et elle est indispensable pour effectuer l'analyse du degré de développement socio-économique des régions au point de vue l'appréciation du degré de la diversification et pour l'élaboration des programmes de développement régional. La classification de NTS divise la Pologne en unités liées territorialement et hiérarchiquement aux cinq niveaux, dont les trois niveaux régionales et deux niveaux locaux. Le concept de région métropolitaine se réduit essentiellement à intégrer la gestion des aires métropolitaines en décentralisant leurs fonctions, leurs pouvoirs et leurs revenus. La métropole devrait être l'unité régionale créée par la loi et qui est semblable avec l'unité de base de la division territoriale de l'État. Ses habitants devraient avoir une influence directe sur le choix des autorités indépendants de communes créant cette entité.

\section{Les modalités de gestion financière}

Le choix de la forme d'organisation et la forme juridique de coopération de villes aura un impact particulier sur la modalité de gestion financière sur l'aire métropolitaine et sur la mobilisation de ressources financières. Parmi les sources existantes on peut indiquer les cotisations et les dotations d'État, ainsi que les fonds européens, les recettes propres (les taxes, les impôts, les bénéfices de la propriété) et les subventions résultant du transfert de tâches par le gouvernement, par la voïvodie et par les communes formant la métropole.

Pour évaluer les règles adoptées en Pologne et le système actuel de financement des missions de service public il faut dire que parmi les solutions proposées, il semble que la solution le plus optimale est la création des districts métropolitains recouvrant les villes ayant droit de district et avec les mêmes tâches. Il faut souligner que le caractère des aires considérées comme les aires métropolitaines est pleinement différencié. Il peut provoquer les difficultés dans l'exercice de la gestion financière uniforme et approprié pour tous. Le législateur, avant de se prononcer sur le régime, devraient examiner quelles tâches doivent être pris en charge par les métropoles et quels sont leurs coûts, pour prévenir la répétition des erreurs déjà commises et que les décisions sur les sources de revenus et les règles de gestion financière n'ont pas été prises comme les dernières. 
L'obligation constitutionnelle de conserver un équilibre entre le montant des revenus des collectivités territoriales et le contenu des tâches, impose au législateur l'obligation de l'appréciation si le niveau global des recettes permet la mise en œuvre des tâches qui sont imposées. L'article 167 de la Constitution ne peut être comprise comme une base pour l'introduction, au profit des collectivités territoriales, l'obligation d'assurance un certain niveau de revenu, indépendamment de la situation financière de l'ensemble du pays. Cette disposition doit être traitée comme une directive fixant les modalités de partage, dans une période donnée, des ressources financières entre le gouvernement central et les administrations locales en tenant compte de ses différents niveaux . La répartition compétente des revenus est importante pour le fonctionnement de l'économie locale, notamment en raison de la diversité du caractère des collectivités territoriales, ainsi que les disproportions du développement.

L'autonomie des collectivités territoriales au niveau de la collecte des recettes se manifeste non seulement dans l'évaluation du montant des recettes provenant de diverses sources, mais aussi dans le droit de poursuivre une politique financière active, dans le cadre de laquelle il y a la possibilité d'emprunter des obligations et l'obligation d'enquête des dettes avec les intérêts pour retard de paiement. La stratégie de la gestion des ressources et le droit de tirer les bénéfices de cette propriété peuvent avoir l'influence sur le niveau des recettes budgétaires. La répartition des recettes entre le public et de l'État et les collectivités territoriales doit se référer à leur mission, et dans d'autres mots - aux frais de l'accomplissement de ces tâches. La redistribution des recettes provenant des impôts par l'intermédiaire des dotations et des subventions ne devrait pas remplacer la division base de sources fiscales et peut être liée à l'existence des sujets beaucoup plus faibles et la nécessité d'influer sur les activités de collectivités territoriales .

La transmission d'une partie des tâches sur la métropole pourrait réduire sensiblement le coût d'exécution, ainsi que d'éliminer la concurrence entre les villes et d'accroître les chances de demande de fonds sur le budget de l'Union européenne et d'autres ressources non-remboursable découlant de sources étrangères. La sélection de l'organisation et de formes juridiques devrait permettre de conserver l'autonomie des villes et d'autre part, de garantir une participation dans les recettes publiques, sans qu'il soit nécessaire la discussion concernant des cotisations de membres et la propriété, les discussions qui peuvent se former dans le cas de la création de l'association communale obligatoire. 


\section{Les conclusions}

La mise en œuvre - en particulier dans l'agglomération silésienne - du district englobant les collectivités territoriales ayant le même statut (les villes ayant droit de district) peuvent être une solution intéressante. Ce district pourrait prendre en charge au moins une partie des tâches de service public qui sont effectuées par les communes avec les ressources de financement, en tenant compte les prix de ces tâches calculés correctement.

Le district, comme une collectivité locale - par opposition aux syndicats des communes - a le droit de posséder ses propres revenus. Il peut obtenir des subventions et des dotations du budget de l'État. Cependant, le système de subventions devrait compenser les recettes des collectivités territoriales de différents niveaux, en prouvant que l'équilibrage est approprié et qu'il prend en compte une capacité financière de l'État et qu'il ne repose pas sur une marge d'appréciation, mais sur les critères objectifs, définis dans la loi .

Cela signifie que la loi doit contenir toutes les dispositions de base pour désigner le type et la nature juridique de diverses sources et toutes les règles concernant la détermination de montant de ce revenu. Ainsi, le niveau global des recettes de collectivités territoriales doit être possible à déterminer, sur la base déjà réglementée par la loi, qui doit maintenir un rang approprié de précision et de détail et ne peut pas être limitée seulement aux dispositions blanchets . En revanche, les subventions ciblées peuvent servir seulement une fonction des revenus complémentaires et ils ne devraient pas être une forme de " soutien » des collectivités territoriales ce qui menace l'autonomie qui est garantie par la Constitution. 


\section{Streszczenie}

Wybór formy organizacyjnej tj. współpracy prawnej jednostek samorządu terytorialnego i przyjętych przez nie zadań, wpływają w znacznym stopniu na zasady zarządzania gospodarką finansową regionów metropolitalnych. Podjęte decyzje przyczyniają się również do wyboru potencjalnych źródeł finansowania tych zadań, przy uwzględnieniu ograniczeń wynikających zarówno z Konstytucji jak i innych ustaw. Wśród sugerowanych rozwiązań znajduje się stworzenie obligatoryjnych stowarzyszeń obejmujących kilka miast lub dzielnic o tym samym statusie prawnym. Każde z tych rozwiązań wymaga przeanalizowania finansowych konsekwencji wprowadzanych regulacji, jak i istniejących instrumentów finansowych oraz ich koordynacji. 